\title{
On the convergence of bumping routes to their limit shapes in the RSK algorithm. Numerical experiments
}

\author{
N. N. Vassilieva,b, PhD, Phys. -Math., Senior Researcher, orcid.org/0000-0002-0841-1168 \\ V. S. Duzhin ${ }^{\mathrm{b}}$, PhD, Phys.-Math., Senior Lecturer, orcid.org/0000-0001-8399-284X, vsduzhin@etu.ru \\ A. D. Kuzmin b, Master Student, orcid.org/0000-0001-9975-4686 \\ a Saint-Petersburg Department of V. A. Steklov Institute of Mathematics of the RAS, 27, Fontanka Emb., \\ 191023, Saint-Petersburg, Russian Federation \\ bSaint-Petersburg Electrotechnical University «LETI», 5, Prof. Popov St., 197376, Saint-Petersburg, \\ Russian Federation
}

\begin{abstract}
Introduction: The Robinson - Schensted - Knuth (RSK) algorithm transforms a sequence of elements of a linearly ordered set into a pair of Young tableaux $P, Q$ of the same shape. This transformation is based on the process of bumping and inserting elements in tableau P according to special rules. The trajectory formed by all the boxes of the tableau P shifted in the RSK algorithm is called the bumping route. D. Romik and P. Śniady in 2016 obtained an explicit formula for the limit shape of the bumping route, which is determined by its first element. However, the rate of convergence of the bumping routes to the limit shape has not been previously investigated either theoretically or by numerical experiments. Purpose: Carrying out computer experiments to study the dynamics of the bumping routes produced by the RSK algorithm on Young tableaux as their sizes increase. Calculation of statistical means and variances of deviations of bumping routes from their limit shapes in $L_{2}$ metric for various values fed to the input of the RSK algorithm. Results: A series of computer experiments have been carried out on Young tableaux consisting of up to 10 million boxes. We used 300 tableaux of each size. Different input values $(0.1,0.3,0.5,0.7,0.9)$ were added to each such tableau using the RSK algorithm, and the deviations of the bumping routes built from these values from the corresponding limit shapes were calculated. Graphs of the statistical mean values and variances of these deviations were produced. It was noticed that the deviations decrease in proportion to the fourth root of the tableau size $n$. An approximation of the dependence of the mean values of deviations on $n$ was obtained using the least squares method.
\end{abstract}

Keywords - RSK algorithm, RSK correspondence, Young diagram, Young tableau, Plancherel measure, Vershik - Kerov curve, bumping route, limit shape, linearly ordered set, numerical experiment, asymptotic combinatorics.

For citation: Vassiliev N. N., Duzhin V. S., Kuzmin A. D. On the convergence of bumping routes to their limit shapes in the RSK algorithm. Numerical experiments. Informatsionno-upravliaiushchie sistemy [Information and Control Systems], 2021, no. 6, pp. 2-9. doi:10.31799/1684-8853-2021-6-2-9

\section{Introduction}

The Robinson - Schensted - Knuth algorithm [1-5], also known as RSK transformation, defines a correspondence between the set of permutations of natural numbers and the set of pairs of standard Young tableaux of the same shape, the so-called insertion tableau $P$ and recording tableau $Q$. The RSK algorithm finds applications in various problems of combinatorics.

The RSK transformation can also be applied to any infinite sequence of a linearly ordered set. In this case, the resulting recording Young tableau is semi-standard. The distinctive feature of such a tableau is that its elements do not decrease in rows and increase in columns. In this article, we use real numbers from the range $[0,1]$ as the input of the RSK algorithm.

The problem of studying the trajectories of bumping routes in the RSK algorithm was first formulated in [6]. Later, the dynamics of motion of an element in the RSK algorithm and the dynamics of tableau $P$ changes began to be studied from the combinatorial point of view in [7-13]. It led to the introduction of a bumping tree and a bumping forest [14], as well as the study of their combinatorial structure. The bumping forest displays all possible transitions when the next sequence entry is added to the Young tableau during the iteration of the RSK algorithm.

An explicit expression for the limit shapes of the bumping routes for arbitrary values of elements inserted in the input sequence is given in [8]. The coordinates of the end of the bumping route give us the position of the added box to the corresponding tableau $P$.

This work is devoted to the study of the convergence of the bumping routes in the tableau $P$ to their limit shapes as the number of boxes in $P$ grows. In the frame of the work, a series of computer experiments were carried out with Young tableaux distributed according to Plancherel measure with sizes up to $10^{7}$ boxes using the developed software package. As a result, the statistical means and variances of the distances between the bumping routes and their limit shapes were obtained for various input values and tableaux sizes.

The developed software package includes a set of operations for working with Young tableaux, an ef- 
ficient implementation of the RSK algorithm, functions for calculating the limit shapes of bumping routes, various generators of random Young tableaux, and much more.

\section{Definitions and basic notations}

Young diagram is a finite set of boxes, arranged in bottom-aligned non-increasing columns. The set of Young diagrams consisting of $n$ boxes is in oneto-one correspondence with the set of integer partitions $n=l_{1}+l_{2}+\ldots+l_{k}$, where $l_{\mathrm{i}}$ are the heights of the columns of a Young diagram, $l_{i} \geq l_{i+1}$. We use so-called French notation to display diagrams, in which rows are horizontal and columns are bottom-aligned.

There used various coordinate systems for visualizing Young diagrams. In this work we use the rectangular coordinate system $(x, y)$ and the Vershik Kerov coordinate system $(u, v)$. The last one can be obtained from the rectangular coordinate system by rotating the axes by $45^{\circ}$. In Fig. $1, a$ and $b$, the bold line shows the shape of the Young diagram $\lambda(4,2,1)$, presented in both coordinate systems.

Young tableau is a Young diagram filled with values which increase in rows and columns. These values can be elements of an arbitrary linearly or- a)

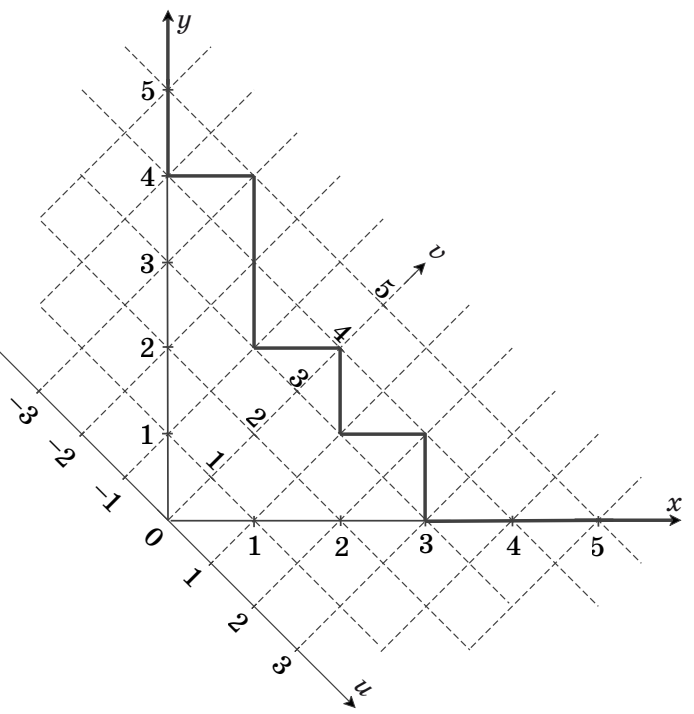

b)

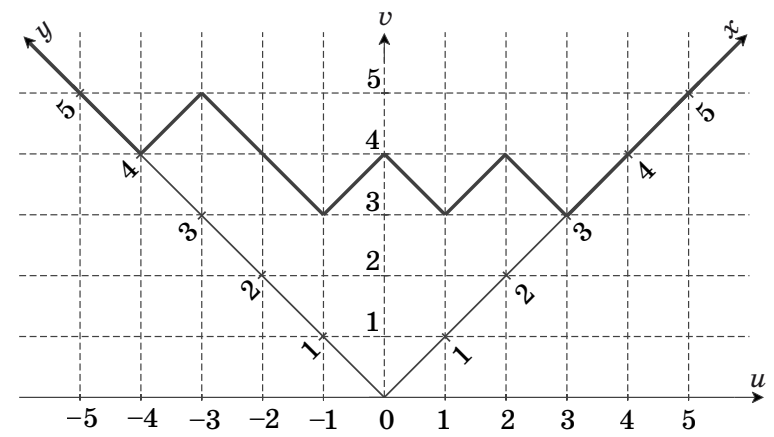

Fig. 1. Young diagram in the rectangular $(a)$ and the Vershik - Kerov $(b)$ coordinate system

a)

\begin{tabular}{|c|c|c|c|c|c|c|c|c|}
\hline 40 & & & & & & & & \\
\hline 34 & & & & & & & & \\
\hline 32 & 47 & & & & & & & \\
\hline 25 & 45 & & & & & & & \\
\hline 23 & 38 & 48 & 50 & & & & & \\
\hline 18 & 33 & 46 & 49 & & & & & \\
\hline 17 & 19 & 28 & 44 & & & & & \\
\hline 11 & 14 & 24 & 35 & 39 & & & & \\
\hline 9 & 10 & 15 & 16 & 21 & 26 & 31 & 42 & 43 \\
\hline 2 & 4 & 6 & 8 & 13 & 2 & 29 & 37 & 41 \\
\hline 1 & 3 & 5 & 7 & 12 & 20 & 27 & 30 & 36 \\
\hline
\end{tabular}

b)

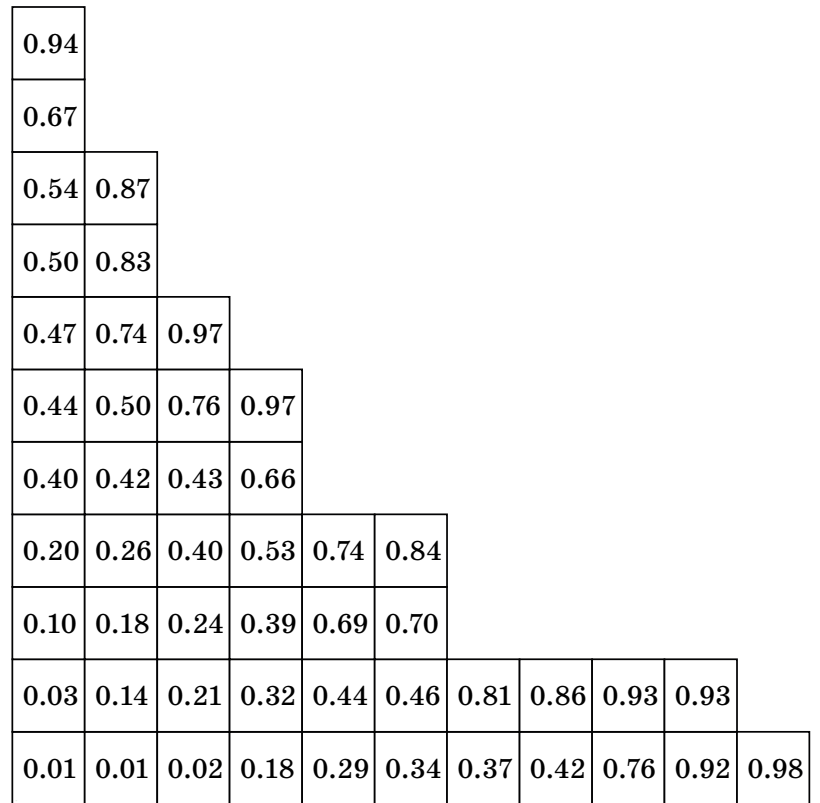

Fig. 2. Two examples of Young tableaux: standard ( $a$ ) and semi-standard $(b)$ tableau 
dered set. In addition to finite Young tableaux, consisting of $n$ boxes, it is possible to consider infinite Young tableaux. It is a mapping $\sigma: \mathbb{Z}_{+}^{2} \Rightarrow \mathbb{N}$ such that for fixed $i, j \in \mathbb{N}$ the values $\sigma_{i, k}$ and $\sigma_{k, j}$ strictly increase as $k \rightarrow \mathbb{N}$. In this case, the mapping $\mathbb{Z}_{+}^{2}$ in the set of natural numbers $\mathbb{N}$ is not necessarily a bijection, since all natural numbers may not be included in the tableau. An alternative name for infinite Young tableaux, used in this context by A. M. Vershik, is integer enumeration of semi-lattice $\mathbb{Z}_{+}^{2}$.

Young tableau is called standard if it is filled with distinct natural numbers 1..n and semi-standard if it is filled with elements of an arbitrary linearly ordered set. In the semi-standard Young tableaux, the values do not decrease by row and strictly increase by column. The examples of a standard and a semi-standard Young tableau are depicted in the Fig. 2, $a$ and $b$.

In this work, we study the properties of insertion of elements into extremely large semi-standard Young tableaux filled by real numbers from the interval $[0,1]$ which are produced by the RSK algorithm.

\section{Robinson - Schensted - Knuth algorithm}

As it was said before, the RSK algorithm transforms an arbitrary input sequence of values into a pair of Young tableaux of the same shape, called insertion tableau $P$ and recording tableau $Q$. In this case, tableau $P$ is a semi-standard tableau filled with inserted values, and tableau $Q$ is a standard tableau filled with natural numbers from 1 to $n$.

Let the input sequence $s_{1}, s_{2}, \ldots, s_{n}$ be fed to the RSK algorithm. We assume that the columns of tableaux $P, Q$ are numbered from left to right, and the rows are numbered from bottom to top. Let us designate the columns of the tableau $P$ as $P_{1}, P_{2}, \ldots$, $P_{n}$, and the element in column col of row $m$ as $P_{c o l, m}$. Initially the tableau $P$ consists of one box containing the element $s_{1}$. Then the algorithm for generating the tableau $P$ is described as follows:

$$
\begin{aligned}
& \text { for each } s_{i} \text { : } \\
& \quad \begin{array}{l}
\text { col }=1 ; \tau=s_{i} ; \\
\text { do } \\
\quad \text { if }\left(\tau>\max \left(P_{c o l}\right)\right) \\
\quad \operatorname{add} \tau \operatorname{above} P_{c o l} ; \\
\text { else } \\
\quad P_{c o l, m}=\min \left(P_{c o l, j}>\tau\right), j \in\left[1 ; \operatorname{height}\left(P_{c o l}\right)\right] \text {; } \\
\quad P_{c o l, m} \leftrightarrows \tau ; \\
k=k+1 ;
\end{array}
\end{aligned}
$$

while $\left(\tau \leq \max \left(P_{c o l}\right)\right)$; end for;

Generation of the tableau $Q$ does not require any additional calculations because after each expansion of the tableau $P$, the value $i$ is placed on the top

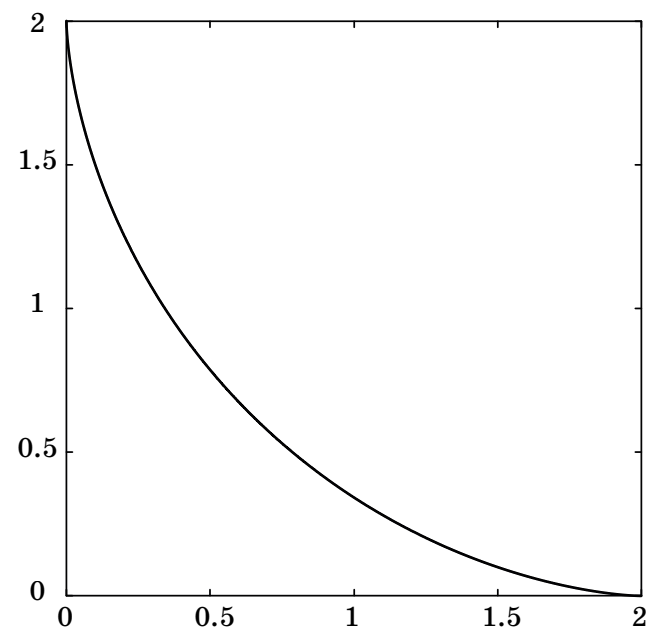

Fig. 3. The Vershik - Kerov limit shape

of the column $Q_{k}$ expanding the shape to match one of tableau $P$.

Note that we start searching for the element $\min \left(P_{c o l, j}>\tau\right)$ from the $m$-th coordinate of the previous bumping in the col-1 column. This is justified by the fact that the value in the box $(\mathrm{col}, \mathrm{m})$ is always greater than $\tau$.

As stated above, the algorithm is capable of processing not only integer or real numbers, but also elements of any linearly ordered set. In this case, the tableau $P$ is filled with elements of this set and therefore in general case is semi-standard.

If the input of the RSK algorithm receives a sequence of random real numbers uniformly distributed on the interval $[0,1]$, then the resulting tableaux will be typical according to the Plancherel measure. Moreover, with an increase in the size of the tableaux, their profile tends towards the Vershik - Kerov curve [15]:

$$
\Omega(u)=\frac{2}{\pi}\left(u \cdot \arcsin \frac{u}{2}+\sqrt{4-u^{2}}\right),|u| \leq 2 .
$$

To transform (1) from the coordinate system $(u$, $v)$ to the coordinate system $(x, y)$, we rotate it by $45^{\circ}$ :

$$
x=\frac{v-u}{2}, y=\frac{v+u}{2} \text {. }
$$

The Vershik - Kerov limit shape in a rectangular coordinate system $(x, y)$ is shown in the Fig. 3 .

Below we will describe the process of inserting some fixed value into an arbitrary insertion tableau $P$.

\section{Bumping routes}

Let us consider a tableau $P$ of the shape $\lambda(n)$ with boxes filled by the values $x_{1}, \ldots, x_{n}$. If we insert an 
element $\alpha$ into the tableau $P$, we get a new tableau $P^{\prime}$ filled with the values $x_{1}, \ldots, x_{n}, \alpha$. This tableau has the shape $\lambda(n+1) \nwarrow \lambda(n)$, i.e. it is obtained from $\lambda(n)$ by adding a box.

To build a tableau $P^{\prime}, \alpha$ is initially placed in the first column, displacing the closest greater value to the second column. The displaced value is put in the second column, "bumping" the next value into the third column, and so on, until the next value is bumped out of the shape $\lambda(n)$.

The sequence of boxes of a tableau $P$, shifted during the process of insertion of a value in the RSK transformation, forms so-called bumping route. An example of a bumping route for a tableau of size 20 is shown in the Fig. 4.

The asymptotic behaviour of the bumping routes was studied in some recent works. Particularly, in [8], the limit shape of the bumping routes depending on the value of the inserted element was studied. The paper [13] was devoted to the investigation of dynamics of concrete values in tableau $P$ during the iterations of the RSK algorithm.

Each bumping route ends with a box at the profile of the corresponding Young diagram. The bumping routes ending in the same box form the so-called bumping tree. The union of all the bumping trees of the tableau called a bumping forest. An example of a Young tableau with the corresponding bumping forest is shown in the Fig. 5. Different bumping trees of the Young tableau are shown in different colours.

In the article [8], the asymptotic behaviour of the bumping routes with an increase in the number of boxes of typical Plancherel tableaux was investigated. Each inserted value of $\alpha$ corresponds to a certain curve, which is the limit shape of the corresponding bumping route.

The formulas below from the paper [8] describe such limit shapes. As mentioned earlier, the limit shapes of Young tableaux, distributed according to the Plancherel measure, after their scaling, is the region bounded by the Vershik - Kerov curve (1). Further, we mean that Young tableaux are scaled in $\sqrt{n}$. So the area under the tableau profile is equal to 1 .

Note that in the current article, the orientation of Young tableaux is changed in comparison with

\begin{tabular}{|c|c|c|c|c|c|}
\hline 11 & 15 & & & & \\
\hline 9 & -13 & 16 & & & \\
\hline 6 & 7 & 14 & -17 & & \\
\hline 3 & 5 & 8 & 12 & 20 & $\rightarrow$ \\
\hline 1 & 2 & 4 & 10 & 18 & 19 \\
\hline
\end{tabular}

- Fig. 4. A bumping route

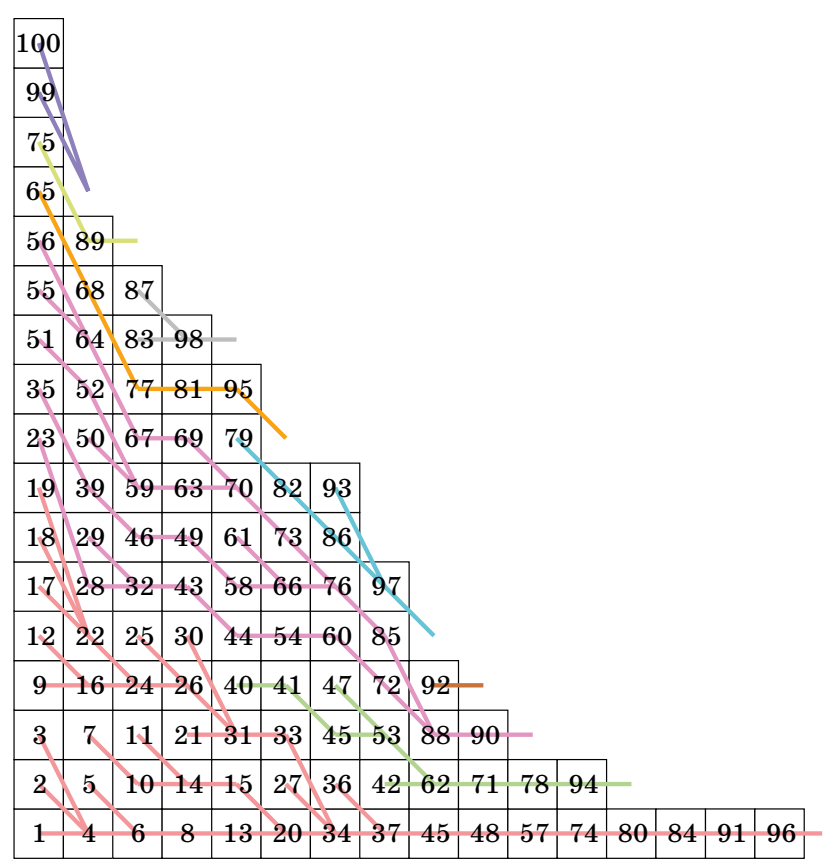

- Fig. 5. Bumping trees of the tableau of size $\mathbf{1 0 0}$

the one used in [8]: we reflect Young tableaux with respect to the bisector $x=y$.

When an element $\alpha \in[0,1]$ is processed by the RSK algorithm, the corresponding bumping route ends in a point with coordinates $u, v$ defined as follows:

$$
\left(u_{\alpha}, v_{\alpha}\right)=\left(F^{-1}(\alpha), \Omega\left(F^{-1}(\alpha)\right)\right),
$$

where $\alpha$ is the value added to the tableau $P ; \Omega$ is the Vershik - Kerov curve (1); $F$ is the distribution function of the Wigner semicircle law:

$$
F(u)=\frac{1}{2}+\frac{1}{\pi}\left(\frac{u \sqrt{4-u^{2}}}{4}+\arcsin \frac{u}{2}\right),|u| \leq 2 .
$$

Let $\alpha$ be the value in the first column from which the bumping route is constructed, and $t$ be some intermediate value on this route. The coordinates $u$ and $v$ in Vershik - Kerov notation for the value $t$ are calculated as follows:

$$
\begin{gathered}
u_{\alpha}(t)=\sqrt{t} \cdot F^{-1}\left(\frac{\alpha}{t}\right), 0 \leq \alpha \leq t \leq 1 ; \\
v_{\alpha}(t)=\sqrt{t} \cdot \Omega\left(F^{-1}\left(\frac{\alpha}{t}\right)\right), 0 \leq \alpha \leq t \leq 1 .
\end{gathered}
$$

To transform $u_{\alpha}(t), v_{\alpha}(t)$ into the rectangular $(x, y)$ coordinate system, we rotate the axis by $45^{\circ}$ counterclockwise:

$$
x_{\alpha}(t)=\frac{v_{\alpha}(t)-u_{\alpha}(t)}{2}
$$




$$
y_{\alpha}(t)=\frac{v_{\alpha}(t)+u_{\alpha}(t)}{2}
$$

Since the end of the bumping route lies on the Vershik - Kerov curve, the value of the $x$ coordinate of the end of the bumping route for some $\alpha$ corresponds to the case $t=1$ in formula (7):

$$
k(\alpha)=x_{\alpha}(1)=\frac{\Omega\left(F^{-1}(\alpha)\right)-F^{-1}(\alpha)}{2} .
$$

Formulas (6), (7) allow to calculate the $x, y$ coordinates for arbitrary intermediate values of $t$ on the bumping route. The function describing the limit shape of the entire route for an arbitrary $\alpha \in[0,1]$ has the form:

$$
\beta_{\alpha}(s)=y_{\alpha}\left(x_{\alpha}^{-1}(s)\right), 0 \leq s \leq k(\alpha),
$$

where $k(\alpha)$ is the coordinate of the projection of the end of the route on the $x$-axis.

The use of formulas (1)-(9) is shown in the Fig. 6. The red line marks the theoretical limit of the bumping route starting from $\alpha$. The parameter $t$ is some intermediate value on this path. Due to the properties of Young tableaux, it satisfies the condition $\alpha \leq t \leq 1$, since the bumping route begins at the element $\alpha$ and ends at the limit shape (1) of the tableau. To determine the coordinates of a box filled by an arbitrary value of $t$ on the bumping route, the limit shape is scaled down by a factor of $\sqrt{t}$, and then the coordinate of the intersection of the bumping route with the resulting curve is calculated, see formulas (4), (5). All the formulas described above are

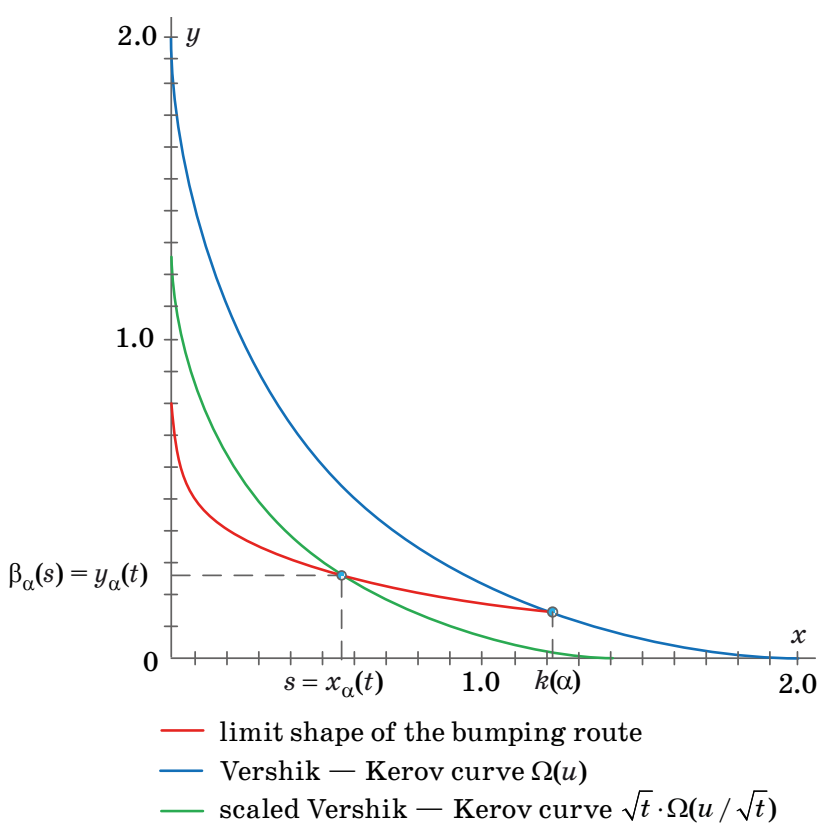

- Fig. 6. Calculation of the coordinates of the limit shape of the bumping route

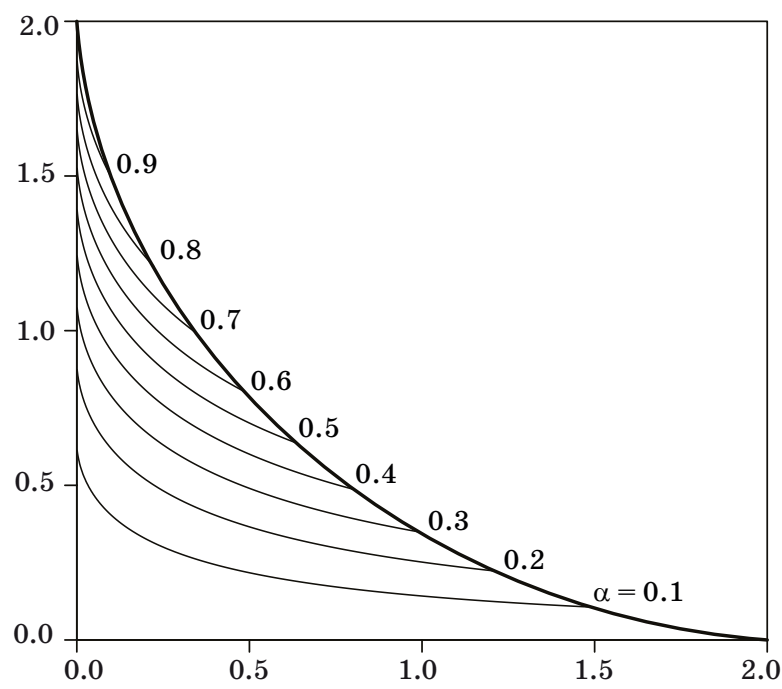

Fig. 7. Limit shapes of the bumping routes

valid for the coordinate system $(u, v)$ and it is possible to transform the obtained coordinates into the rectangular coordinate system $(x, y)$ using formulas (6), (7). The limit shapes of the bumping routes for various inserted values of $\alpha$ are shown in the Fig. 7 .

Note that in the case of $\alpha=1$, the value is placed on the top of the first column and its bumping route includes only the box with this value; in the case of $\alpha=0$, the bumping route goes along the entire first row.

There are many possible ways to implement the RSK algorithm. In the framework of this work, the standard implementation and the implementation using the bumping forest were made. In the first case, for each bumped value, we search for the closest greater value in the next column. In the second case, we do not need to perform such a search because the algorithm maintains the correctness of the bumping forest structure in tableau $P$.

\section{Numerical experiments}

The goal of this work is to investigate the convergence of the bumping routes in the $L_{2}$ norm using numerical experiments. In the course of these experiments, typical Plancherel-distributed Young tableaux were constructed with sizes up to $n=10^{7}$ boxes, for which the means and variances of the deviations of the bumping routes from the limit shape were calculated. Since our computer experiments were carried out on extra-large tableaux, they required significant computational resources. Therefore, for each $n$, we limited ourselves to generating 300 tableaux only.

Note that the different bumping routes corresponding to the same value of $\alpha$ could be end- 
ed in different boxes of the profile of the tableau. Therefore, when comparing these routes, we scale them along the $x$-axis so that their projections on the $x$-axis coincide with the projection on the $x$-axis of the corresponding limit shape.

A certain fixed value of $\alpha$ was inserted into each of the generated tableaux $P$, the corresponding bumping route was constructed, and its deviation from the limit shape in $L_{2}$ norm was calculated. Then the statistical means and variances were calculated for 300 obtained deviations. As a result, for each pair of values of $n$, $\alpha$, we obtained the mean value and the variance of deviation of bumping routes from the limit shape.

\section{Experimental results}

In our computational experiments, we have constructed Young tableaux with sizes $n=10^{5} . .10^{7}$ boxes with a step of $10^{5}$ for which the means and variances of the deviations of the bumping routes from the limit shape were calculated. The following values of the element $\alpha$ were fed to the input of the RSK algorithm: $\alpha=0.1,0.3,0.5,0.7,0.9$.

It was noticed that the distances between the bumping routes and the corresponding limit curves decrease proportionally $n^{-\frac{1}{4}}$. Therefore, these distances were approximated by the following curve using the least squares method:

$$
f(n)=a \cdot n^{-\frac{1}{4}}+b \cdot n^{-\frac{1}{2}} .
$$

We calculated the coefficient $b$ to estimate the remainder term, which is small in comparison with $-\frac{1}{4}$ $n^{-\frac{1}{4}}$. Coefficients $a(\alpha)$ and $b(\alpha)$, obtained for tableaux of sizes $n \in\left[10^{5} . .10^{7}\right]$, are shown in the Table.

The graphs of the deviations of the bumping routes from the limit shape for $\alpha=0.1,0.3,0.5,0.7$,

Values of the coefficients $a$ and $b$ depending on inserted values of $\alpha$

\begin{tabular}{|c|c|c|}
\hline$\alpha$ & $a$ & $b$ \\
\hline 0.1 & 0.280062 & 0.358644 \\
\hline 0.3 & 0.422171 & 0.544981 \\
\hline 0.5 & 0.511865 & 0.661715 \\
\hline 0.7 & 0.585168 & 0.167618 \\
\hline 0.9 & 0.606942 & 0.760016 \\
\hline
\end{tabular}

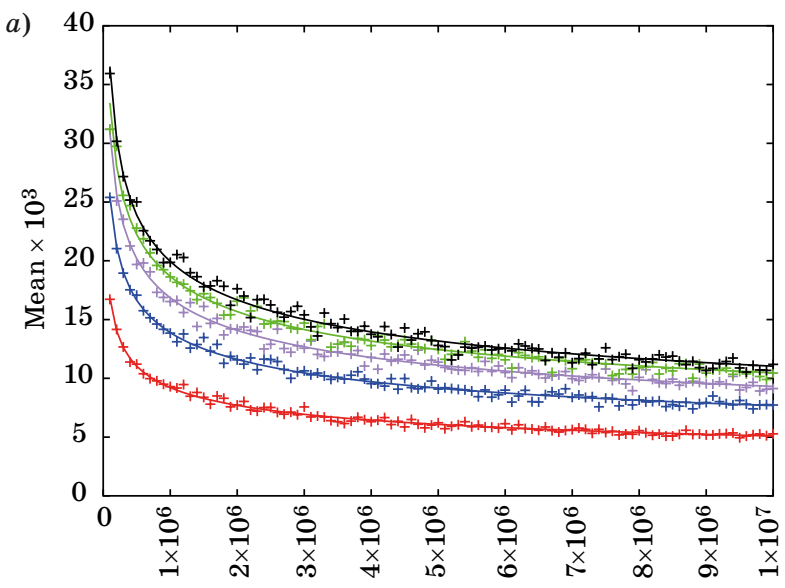

Tableau size

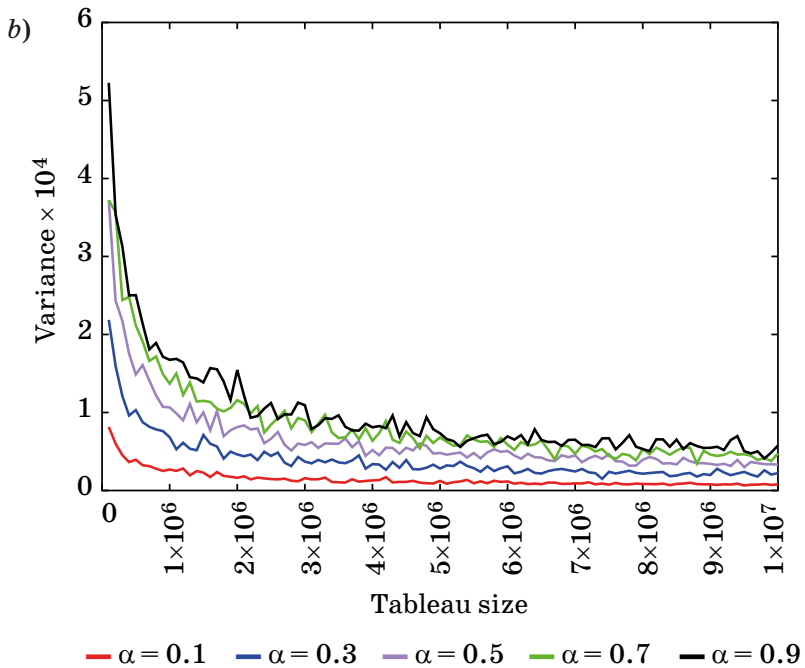

- Fig. 8. Mean values $(a)$ and variances $(b)$ of deviations of bumping routes from their limit shapes and corresponding approximating curves

0.9 are shown in the Fig. 8, $a$. For each tableau size $<10^{6}, 1000$ tableaux and the corresponding routes were built. For larger sizes, 300 tableaux were built for each size.

With the increase in the size of the tableaux, deviations from the limit shape gradually decrease. In addition to the experimentally obtained values, the Fig. 8, $a$ shows approximating curves (10) constructed using the values from the Table. As we see from the figure, the curves approximate the experimental data quite well.

Figure $8, b$ shows the variances of the obtained deviations for the same five values of $\alpha$. We did not attempt to approximate the variance graphs, but we assume that it can also be done using function similar to (10). As one can see from the figure, the variance, although slowly, but decreases with the increase in the size of the tableaux. The smaller the $\alpha$ values, the smaller the values and oscillations of variances. 


\section{Conclusions}

We performed computer experiments to study the rate of convergence of the bumping routes of the RSK algorithm to the limiting theoretical curves obtained in [8] for different inserted values $\alpha$.

300 Plancherel-distributed Young tableaux were generated for each size $n=10^{5} . .10^{7}$ with a step of $10^{5}$. Next, 5 values of $\alpha=0.1,0.3,0.5,0.7,0.9$ have been added to these tableaux using the RSK algorithm. Therefore, we obtained the bumping routes for each inserted $\alpha$. For each tableau, the deviation of the bumping route from the corresponding limit shape in the $L_{2}$ norm was calculated. The means and variances of the calculated deviations were obtained. It was noticed that these mean values decrease with increasing size in proportion to $n^{-\frac{1}{4}}$. The graphs of the mean values were approximat-

\section{References}

1. Robinson G. de B. On the representations of the symmetric group. American Journal of Math., 1938, vol. 60 , pp. $745-760$.

2. Schensted C. Longest increasing and decreasing subsequences. Canadian Journal of Math., 1961, vol. 13, pp. 179-191.

3. Knuth D. E. Permutations, matrices, and generalized Young tableaux. Pacific J. Math., 1970, vol. 34, iss. 3, pp. 709-727.

4. Stanley R. P. Enumerative combinatorics. Ser. Cambridge Studies in Advanced Mathematics, vol. 62 . Cambridge University Press, Cambridge, 1999. Vol. 2. $581 \mathrm{p}$.

5. Fulton W. Young Diagrams, with applications to representation theory and geometry. Cambridge University Press, 1996. 272 p.

6. Moore C. Flows in Young diagrams. 2006. Available: http://tuvalu.santafe.edu/ moore/gallery.html (accessed 18 August 2021).

7. Vershik A. M. Combinatorial encoding of Bernoulli schemes and the asymptotic behavior of Young tableaux. Funct Anal Its Appl, 2020, vol. 54, pp. 77-92. https://doi.org/10.1134/S001626632002001X

8. Romik D., Sniady P. Limit shapes of bumping routes in the Robinson - Schensted correspondence. Random Struct.Algorithms, 2016, vol. 48, iss. 1, pp. 171182. https://doi.org/10.1002/rsa.20570

9. Marciniak M., Maślanka Ł. \& Śniady P. Poisson limit of bumping routes in the Robinson - Schensted cor- ed by the least squares method using a function $f(n)=a \cdot n^{-\frac{1}{4}}+b \cdot n^{-\frac{1}{2}}$

Unfortunately, the slow rate of convergence of the bumping routes to the limit shape, as well as the limited computational resources, did not allow us to increase the number and the sizes of the Plancherel tableaux involved into our experiments.

Further plans include the study of the distance between the coordinates of some fixed value lying on the bumping route and on the corresponding limit shape. We also plan to optimize the developed code in order to process larger Young tableaux.

\section{Financial support}

This work was supported by an RNF grant 2111-00152. respondence. Probab. Theory Relat. Fields, 2021, vol. 181, pp. 1053-1103. https://doi.org/10.1007/ s00440-021-01084-y

10. Marciniak M. Hydrodynamic limit of the RobinsonSchensted - Knuth algorithm. Random Struct. Algorithms, 2021. https://doi.org/10.1002/rsa.21016

11. Śniady P. Robinson - Schensted - Knuth algorithm, jeu de taquin and Kerov - Vershik measures on infinite tableaux. SIAM Journal on Discrete Mathematics, 2013, vol. 28, iss. 2. doi:10.1137/130930169

12. Vassiliev N. N., Duzhin V. S., Kuzmin A. D. Investigation of properties of equivalence classes of permutations by inverse Robinson - Schensted - Knuth transformation. Informatsionno-upravliaiushchie sistemy [Information and Control Systems], 2019, no. 1, pp. 11-22 (In Russian). doi:10.31799/1684-8853-20191-11-22

13. Duzhin V. S. Investigation of insertion tableau evolution in the Robinson - Schensted - Knuth correspondence. Discr. Contin. Models Appl. Comput. Sci., 2019, vol. 27, iss. 4, pp. 316-324.

14. Duzhin V., Kuzmin A., Vassiliev N. RSK bumping trees and a fast RSK algorithm. International Conference Polynomial Computer Algebra, Saint-Petersburg, April 15-20, 2019, 2019, pp. 64-66.

15. Vershik A. M., and Kerov S. V. Asymptotic of the largest and the typical dimensions of irreducible representations of a symmetric group. Functional Anal. Appl., 1985, vol. 19, iss. 1, pp. 21-31. 
УДК $519.165,517.938$

doi:10.31799/1684-8853-2021-6-2-9

О сходимости путей выталкиваний в алгоритме RSK к их предельной форме. Численные эксперименты

Н. Н. Васильев ${ }^{a, \sigma}$, канд. физ.-мат. наук, старший научный сотрудник, orcid.org/0000-0002-0841-1168

В. С. Дужин ${ }^{\sigma}$, канд. физ.-мат. наук, старший преподаватель, orcid.org/0000-0001-8399-284X, vsduzhin@etu.ru

А. Д. Кузьмин ${ }^{\sigma}$, магистрант, orcid.org/0000-0001-9975-4686

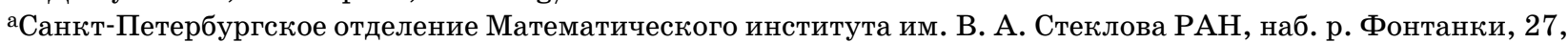
Санкт-Петербург, 191023, РФ

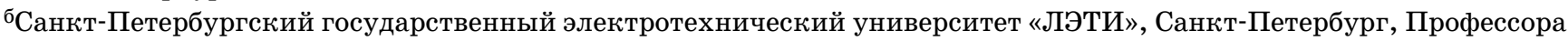
Попова ул., 5, Санкт-Петербург, 197376, РФ

Введение: алгоритм Робинсона - Шенстеда - Кнута (RSK) преобразует последовательность элементов линейно упорядоченного множества в пару таблиц Юнга $P, Q$ одинаковой формы. Это преобразование основано на выталкивании и вставке элементов в таблице $P$ по специальным правилам. Траектория, которую образуют последовательно выталкиваемые клетки таблицы $P$ в алгоритме RSK, называется путем выталкиваний. Явная формула предельной формы пути выталкиваний, которая определяется его начальным элементом, была получена Д. Ромиком и П. Сняды в 2016 г. Однако скорость сходимости путей выталкиваний к предельной форме ранее не исследовалась ни теоретически, ни с помощью численных экспериментов. Цель: проведение компьютерных экспериментов в целях изучения динамики путей выталкиваний, порождаемых алгоритмом RSK в таблицах Юнга, с ростом их размера; вычисление статистических средних и дисперсий отклонений путем выталкиваний от их предельных форм в метрике $L_{2}$ для различных значений, подаваемых на вход алгоритма RSK. Результаты: проведена серия компьютерных экспериментов над таблицами Юнга размера до 10 млн клеток. Использовалось по 300 таблиц каждого размера. В каждую такую таблицу с помощью алгоритма RSK добавлялись различные входные значения $(0,1 ; 0,3 ; 0,5 ; 0,7 ; 0,9)$ и были вычислены отклонения путей выталкиваний, построенных от этих значений, от соответствующих предельных форм. Построены графики средних значений и дисперсий этих отклонений. Замечено, что отклонения убывают пропорционально корню четвертой степени из размера таблицы $n$. Получена аппроксимация зависимости средних значений отклонений от $n$ с помощью метода наименьших квадратов.

Ключевые слова - алгоритм RSK, соответствие RSK, диаграмма Юнга, таблица Юнга, мера Планшереля, кривая Вершика Керова, путь выталкиваний, предельная форма, линейно упорядоченное множество, численный эксперимент, асимптотическая комбинаторика.

Для цитирования: Vassiliev N. N., Duzhin V. S., Kuzmin A. D. On the convergence of bumping routes to their limit shapes in the RSK algorithm. Numerical experiments. Информационно-управляющие системы, 2021, № 6, с. 2-9. doi:10.31799/1684-8853-2021-6-2-9 For citation: Vassiliev N. N., Duzhin V. S., Kuzmin A. D. On the convergence of bumping routes to their limit shapes in the RSK algorithm. Numerical experiments. Informatsionno-upravliaiushchie sistemy [Information and Control Systems], 2021, no. 6, pp. 2-9. doi:10.31799/1684-8853-2021-6-2-9

Финансовая поддержка

Работа поддержана грантом РНФ № 21-11-00152. 